\title{
Solubility of Anthracene in Binary Diisopropyl Ether + Alkane Solvent Mixtures at $298.15 \mathrm{~K}$
}

\author{
Miranda Carrillo, Monica Corella, Kimberle Wolcott, Kaci R. Bowen, and William E. Acree, Jr.*
}

Department of Chemistry, P.O. Box 305070, University of North Texas, Denton, Texas 76203-5070

\begin{abstract}
Experimental solubilities are reported for anthracene in six binary diisopropyl ether + alkane solvent mixtures at 298.15 K. The alkane solvents studied were hexane, heptane, octane, cyclohexane, methylcyclohexane, and 2,2,4trimethylpentane. Results of these measurements were used to test a mathematical representation based on the combined nearly ideal binary solvent (NIBS)/Redlich-Kister equation. For the six systems studied, the combined NIBS/Redlich-Kister equation was found to accurately describe the experimental data, with an average absolute deviation between measured and back-calculated values being approximately $\pm 0.3 \%$.
\end{abstract}

\section{Introduction}

Solid-liquid equilibrium data for crystalline polycyclic aromatic hydrocarbons dissolved in organic solvents are becoming increasingly important in the petroleum industry, particularly in light of present trends toward heavier feedstocks and the known (or suspected) carcinogenicity/mutagenicity of many larger polycyclic aromatic hydrocarbons. Over the past 20 years, we have reported experimental solubility data for anthracene and pyrene dissolved in numerous binary solvents and have developed a fairly simple predictive method for estimating the solubility of crystalline organic compounds in ternary ${ }^{1-4}$

$$
\begin{array}{r}
\ln x_{\mathrm{A}}^{\mathrm{sat}}=x_{\mathrm{B}}^{\mathrm{o}} \ln \left(x_{\mathrm{A}}^{\mathrm{sat}}\right)_{\mathrm{B}}+x_{\mathrm{C}}^{\mathrm{o}} \ln \left(x_{\mathrm{A}}^{\mathrm{sat}}\right)_{\mathrm{C}}+x_{\mathrm{D}}^{\mathrm{o}} \ln \left(x_{\mathrm{A}}^{\mathrm{sat}}\right)_{\mathrm{D}}+ \\
x_{\mathrm{B}}^{\mathrm{o}} x_{\mathrm{C}}^{\mathrm{o}} \sum_{i=0}^{r} S_{\mathrm{BC}, i}\left(x_{\mathrm{B}}^{\mathrm{o}}-x_{\mathrm{C}}^{\mathrm{o}}\right)^{i}+x_{\mathrm{B}}^{\mathrm{o}} x_{\mathrm{D}}^{\mathrm{o}} \sum_{j=0}^{s} S_{\mathrm{BD}, j}\left(x_{\mathrm{B}}^{\mathrm{o}}-x_{\mathrm{D}}^{\mathrm{o}}\right)^{j}+ \\
x_{\mathrm{C}}^{\mathrm{o}} x_{\mathrm{D}}^{\mathrm{o}} \sum_{k=0}^{t} S_{\mathrm{CD}, k}\left(x_{\mathrm{C}}^{\mathrm{o}}-x_{\mathrm{D}}^{\mathrm{o}}\right)^{k}
\end{array}
$$

and higher-order multicomponent solvent mixtures ${ }^{5}$

$$
\ln x_{\mathrm{A}}^{\mathrm{sat}}=\sum_{I} x_{I}^{\mathrm{o}} \ln \left(x_{\mathrm{A}}^{\mathrm{sat}}\right)_{I}+\sum_{I} \sum_{J>I}\left(x_{I}^{\mathrm{o}} x_{J}^{\mathrm{o}} \sum_{i=0}^{r} S_{I J, i}\left(x_{I}^{\mathrm{o}}-x_{J}^{\mathrm{o}}\right)^{i}\right)
$$

based on the extended form Combined Nearly Ideal Binary Solvent (NIBS)/Redlich-Kister solution model. Predictions are based on the measured solubility data in all of the contributing sub-binary solvent mixtures. In eqs 1 and $2 x_{I}^{\mathrm{o}}$ values refer to the initial mole fraction solvent composition of component $i$ calculated as if the solute were not present, and $\left(x_{\mathrm{A}}^{\mathrm{sat}}\right)_{I}$ denotes the measured solute solubility in pure solvent $i$. The various $S_{I J, i}$ parameters can be evaluated with a least-squares regression analysis.

In the present study, we extend our solubility measurements to binary solvent mixtures containing diisopropyl ether. Experimental solubilities are reported for anthracene dissolved in six binary mixtures containing diisopropyl ether with hexane, heptane, octane, cyclohexane, methylcyclohexane, and 2,2,4trimethylpentane at $298.15 \mathrm{~K}$. Results of these measurements

\footnotetext{
*Corresponding author. E-mail: acree@unt.edu.
}

are used to calculate the $S_{I J, i}$ curve-fit coefficients. To date, the solubility behavior of anthracene in binary solvent mixtures containing diisopropyl ether has not been studied.

\section{Experimental Methods}

Materials. Anthracene (Aldrich, 99+ \%) was recrystallized several times from 2-propanone to yield a purified sample having a melting point temperature of $T / \mathrm{K}=489$. Diisopropyl ether (Aldrich, $99 \%$, anhydrous), hexane (Aldrich, $99 \%$ ), heptane (Aldrich, $99 \%$, anhydrous), octane (Aldrich, 99+\%, anhydrous), cyclohexane (Aldrich, HPLC grade, 99.9+ \%), methylcyclohexane (Aldrich, 99+\%, anhydrous), and 2,2,4trimethylpentane (Aldrich, HPLC Grade, $99.7 \%$ ) were stored over molecular sieves and distilled shortly before use. Gas chromatographic analysis showed the solvent mole fraction purities to be $99.7 \%$ or better. Binary solvent mixtures were prepared by mass so that composition could be calculated to 0.0001 mole fraction.

Apparatus and Procedure. Excess solute and solvent were placed in amber glass bottles and allowed to equilibrate in a constant temperature water bath at $T / \mathrm{K}=298.15 \pm 0.05$ for at least 3 days (often longer) with periodic shaking in order to facilitate dissolution of the solid. Attainment of equilibrium was verified by repetitive measurements after a minimum of 3 additional days and by approaching equilibrium from supersaturation by pre-equilibrating the solutions at a slightly higher temperature. Aliquots of saturated anthracene solutions were transferred through a coarse filter into a tared volumetric flask to determine the amount of sample and then diluted quantitatively with methanol for spectrophometric analysis at $356 \mathrm{~nm}$ on a Bausch and Lomb Spectronic 2000. Concentrations of the dilute solutions were determined from a Beer-Lambert law absorbance versus concentration working curve. Molar absorptivities of the nine standard solutions systematically with molar concentration and ranged from $\epsilon /\left(\mathrm{L} \cdot \mathrm{mol}^{-1} \cdot \mathrm{cm}^{-1}\right)=7450$ to $\epsilon /\left(\mathrm{L} \cdot \mathrm{mol}^{-1} \cdot \mathrm{cm}^{-1}\right)=7150$ for anthracene compositions ranging from $C /\left(\mathrm{mol} \cdot \mathrm{L}^{-1}\right)=6.75 \times 10^{-5}$ to $C /\left(\mathrm{mol} \cdot \mathrm{L}^{-1}\right)=2.25 \times$ $10^{-4}$. Identical molar absorptivities were obtained for select anthracene standard solutions that contained volume fractions up to $5 \%$ of the neat diisopropyl ether and alkane cosolvents. Experimental molar concentrations were converted to mass fraction solubility by multiplying by the molar mass of 
Table 1. Experimental Mole Fraction Solubilities $\left(x_{\mathrm{A}}^{\text {sat }}\right)$ of Anthracene in Binary Diisopropyl Ether (B) + Alkane (C) Solvent Mixtures at 298.15 K

\begin{tabular}{|c|c|c|c|}
\hline$x_{\mathrm{B}}^{\mathrm{o}}$ & $x_{\mathrm{A}}^{\mathrm{sat}}$ & $x_{\mathrm{B}}^{\mathrm{o}}$ & $x_{\mathrm{A}}^{\mathrm{sat}}$ \\
\hline \multicolumn{4}{|c|}{ Diisopropyl Ether (B) + Hexane (C) } \\
\hline 0.0000 & 0.001274 & 0.5841 & 0.001980 \\
\hline 0.1098 & 0.001463 & 0.7732 & 0.002238 \\
\hline 0.2022 & 0.001570 & 0.8892 & 0.002419 \\
\hline 0.3817 & 0.001756 & 1.0000 & 0.002515 \\
\hline 0.4835 & 0.001877 & & \\
\hline \multicolumn{4}{|c|}{ Diisopropyl Ether (B) + Heptane (C) } \\
\hline 0.0000 & 0.001576 & 0.6102 & 0.002180 \\
\hline 0.1357 & 0.001711 & 0.7970 & 0.002337 \\
\hline 0.2247 & 0.001799 & 0.8930 & 0.002430 \\
\hline 0.4129 & 0.001981 & & \\
\hline 0.5041 & 0.002074 & & \\
\hline \multicolumn{4}{|c|}{ Diisopropyl Ether (B) + Octane (C) } \\
\hline 0.0000 & 0.001838 & 0.6317 & 0.002383 \\
\hline 0.1401 & 0.001991 & 0.8188 & 0.002504 \\
\hline 0.2374 & 0.002064 & 0.8840 & 0.002533 \\
\hline 0.4258 & 0.002202 & & \\
\hline 0.5378 & 0.002311 & & \\
\hline \multicolumn{4}{|c|}{ Diisopropyl Ether (B) + Cyclohexane (C) } \\
\hline 0.0000 & 0.001553 & 0.4356 & 0.002213 \\
\hline 0.1004 & 0.001768 & 0.5831 & 0.002313 \\
\hline 0.1502 & 0.001867 & 0.7577 & 0.002435 \\
\hline 0.3444 & 0.002145 & 0.8688 & 0.002480 \\
\hline 0.4344 & 0.002202 & & \\
\hline \multicolumn{4}{|c|}{ Diisopropyl Ether (B) + Methylcyclohexane (C) } \\
\hline 0.0000 & 0.001649 & 0.4754 & 0.002296 \\
\hline 0.0990 & 0.001860 & 0.7635 & 0.002496 \\
\hline 0.1945 & 0.002020 & 0.8924 & 0.002520 \\
\hline 0.2898 & 0.002121 & & \\
\hline 0.3721 & 0.002182 & & \\
\hline \multicolumn{4}{|c|}{ Diisopropyl Ether (B) + 2,2,4-Trimethylpentane (C) } \\
\hline 0.0000 & 0.001074 & 0.6343 & 0.001897 \\
\hline 0.1349 & 0.001229 & 0.8184 & 0.002219 \\
\hline 0.2408 & 0.001356 & 0.9086 & 0.002380 \\
\hline 0.4530 & 0.001627 & & \\
\hline 0.5410 & 0.001752 & & \\
\hline
\end{tabular}

anthracene, volume(s) of the volumetric flask(s) used, and any dilutions required to place the measured absorbances on the Beer-Lambert law absorbance versus concentration working curve and then dividing by the mass of the saturated solution analyzed. Mole fraction solubilities were computed from mass fraction solubility using the binary solvent initial mole fraction compositions and molar masses of the solute and both cosolvents. Experimental anathracene solubilities in the six binary diisopropyl ether + alkane mixtures studied are listed in Table 1. Numerical values represent the average of between four and eight independent determinations, with the measured mole fraction solubilities being reproducible to within $\pm 1.5 \%$. Calculated standard deviations ranged from $\sigma_{n-1}=0.000020$ to $\sigma_{n-1}=0.000035$ mole fraction.

\section{Results and Discussion}

For a binary solvent mixture, eq 2 takes the mathematical form of

$$
\begin{aligned}
\ln x_{\mathrm{A}}^{\mathrm{sat}}=x_{\mathrm{B}}^{\mathrm{o}} \ln \left(x_{\mathrm{A}}^{\mathrm{sat}}\right)_{\mathrm{B}}+x_{\mathrm{C}}^{\mathrm{o}} \ln \left(x_{\mathrm{A}}^{\mathrm{sat}}\right)_{\mathrm{C}}+ & \\
& x_{\mathrm{B}}^{\mathrm{o}} x_{\mathrm{C}}^{\mathrm{o}} \sum_{i=0}^{r} S_{\mathrm{BC}, i}\left(x_{\mathrm{B}}^{\mathrm{o}}-x_{\mathrm{C}}^{\mathrm{o}}\right)^{i}
\end{aligned}
$$

The ability of eq 3 to represent mathematically the experimental solubility of anthracene in the six binary diisopropyl ether + alkane solvent mixtures is summarized in Table 2 in the form of the "curve-fit" parameters and the average absolute percent
Table 2. Mathematical Representation of Anthracene Solubilities in Several Binary Diisopropyl Ether (B) + Alkane (C) Solvent Mixtures Based on Equation 3

\begin{tabular}{lcc}
\hline \multicolumn{1}{c}{ solvent $(\mathrm{B})+$ solvent $(\mathrm{C})$} & \multicolumn{1}{c}{$S_{\mathrm{i}}^{\mathrm{a}}$} & $100 \times \mathrm{dev}^{b}$ \\
\hline diisopropyl ether + hexane & 0.195 & 0.3 \\
& -0.177 & \\
& 0.490 & \\
diisopropyl ether + heptane & $0.153^{c}$ & 0.2 \\
diisopropyl ether + octane & 0.216 & 0.3 \\
& 0.084 & \\
& 0.258 & \\
diisopropyl ether + cyclohexane & 0.546 & 0.3 \\
& -0.303 & \\
diisopropyl ether + methylcyclohexane & 0.205 & \\
& 0.494 & 0.4 \\
& -0.219 & \\
diisopropyl ether + 2,2,4-trimethylpentane & 0.325 & \\
& 0.113 & 0.1 \\
& 0.036 & \\
& 0.177 &
\end{tabular}

${ }^{a}$ Curve-fit coefficients are ordered as $S_{0}, S_{1}$, and $S_{2} \cdot{ }^{b}$ Deviation $=$ $\Sigma\left|\left[\left(x_{\mathrm{A}}^{\text {sat }}\right)^{\text {calc }}-\left(x_{\mathrm{A}}^{\text {sat }}\right)^{\exp }\right] /\left(x_{\mathrm{A}}^{\text {sat }}\right)^{\exp }\right| / N .{ }^{c}$ Only a single curve-fit coefficient was needed.

deviations in back-calculated solubilities. The parameters $S$ were computed from a polynomial least-squares analysis of

$$
\begin{aligned}
& {\left[\ln x_{\mathrm{A}}^{\mathrm{sat}}-x_{\mathrm{B}}^{\mathrm{o}} \ln \left(x_{\mathrm{A}}^{\mathrm{sat}}\right)_{\mathrm{B}}-x_{\mathrm{C}}^{\mathrm{o}} \ln \left(x_{\mathrm{A}}^{\mathrm{sat}}\right)_{\mathrm{C}}\right] /\left(x_{\mathrm{B}}^{\mathrm{o}} x_{\mathrm{C}}^{\mathrm{o}}\right)=} \\
& S_{0}+S_{1}\left(x_{\mathrm{B}}^{\mathrm{o}}-x_{\mathrm{C}}^{\mathrm{o}}\right)+S_{2}\left(x_{\mathrm{B}}^{\mathrm{o}}-x_{\mathrm{C}}^{\mathrm{o}}\right)^{2}
\end{aligned}
$$

using the commercial software TableCurve (Janel Scientific, Corte Madera, CA). Careful examination of the Table 2 reveals that eq 3 does provide an accurate mathematical representation for how the solubility of anthracene varies with solvent composition. The overall average absolute deviation between back-calculated and experimental values is $\pm 0.3 \%$, which is less than the uncertainty associated with the measured mole fraction solubilities.

\section{Literature Cited}

(1) Deng, T.; Acree, W. E., Jr. Solubility of anthracene in ternary propanol + 2,2,4-trimethylpentane + cyclohexane and butanol $+2,2,4-$ trimethylpentane + cyclohexane solvent mixtures. J. Chem. Eng. Data 1998, 43, 1059-1061.

(2) Deng, T.; Hernández, C. E.; Roy, L. E.; Acree, W. E., Jr. Solubility of anthracene in ternary (propanol + heptane + cyclohexane) and (butanol + heptane + cyclohexane) solvent mixtures. J. Chem. Thermodyn. 1999, 31, 205-210.

(3) Deng, T.; Acree, W. E., Jr. Solubility of anthracene in ternary propanol + butanol + cyclohexane solvent mixtures. J. Chem. Eng. Data 1998, $43,1062-1064$

(4) Debase, E. M.; Acree, W. E., Jr. Solubility of pyrene in ternary propanol + butanol + cyclohexane solvent mixtures at $299.15 \mathrm{~K} . J$. Chem. Eng. Data 2001, 46, 991-993.

(5) Deng, T.; Horiuchi, S.; De Fina, K. M.; Hernández, C. E.; Acree, W. E. Jr. Solubility of anthracene in multicomponent solvent mixtures containing propanol, butanol and alkanes. J. Chem. Eng. Data 1999, $44,798-802$.

Received for review August 27, 2006. Accepted October 5, 2006. The authors thank the University of North Texas Research Council for partial support of this work. M.C., M.C., and K.W. thank the U.S. Department of Education for the support provided to them under the Upward Bound Math and Science Program. K.R.B. thanks the U.S. Department of Education for support provided under the Ronald E. McNair Postbaccalaureate Achievement Program.

JE060380P 